\title{
A New Era of Intraoperative Neuromonitoring: Beyond the Electromyography Endotracheal Tube During Thyroid Surgery
}

\author{
Sung-Chan Shin (iD) B Byung-Joo Lee (i] \\ Department of Otorhinolaryngology, Head and Neck Surgery, Pusan National University School of Medicine, Pusan National University and \\ Biomedical Research Institute, Pusan National University Hospital, Busan, Korea
}

It is important to preserve the recurrent laryngeal nerve (RLN) during thyroidectomy. Although the RLN can be preserved by visual identification, intraoperative neuromonitoring (IONM) systems using an electromyography (EMG) endotracheal tube with a surface electrode have been developed to identify the functional integrity of the RLN during surgery. These systems help to check the status of the RLN by measuring the EMG signal of the vocalis muscle after weak electrical stimulation is applied to the RLN via a nerve stimulator. Recent studies have revealed that IONM reduces permanent and temporary RLN injuries during thyroidectomy [1,2].

An IONM system consists of a recording side and a stimulating side. The recording side detects muscle movements using EMG. The stimulating side provides weak electrical stimulation to the nerve via a nerve stimulator. Even if IONM is used during thyroidectomy, stimulating the nerve is necessary to confirm whether a nerve injury has occurred. However, repeated stimulation of the nerve during surgery is cumbersome and inefficient. Therefore, methods using a stimulating dissecting instrument or an attachable nerve stimulator capable of stimulating the RLN simultaneously with surgical dissection during open and remote-access thyroidectomy are being developed [3-5].

The most important problem with IONM using an EMG endotracheal tube as the recording side is that its positive predictive value is relatively low, ranging widely from about $10 \%$ to $90 \%$, compared to its high negative predictive value, which ranges from $92 \%$ to $100 \%$ [6]. This is because the contact between the electrodes of the EMG endotracheal tube and the vocal folds is affected by the insertion direction or displacement of the EMG endotracheal tube and the patient's postural changes during surgery. Another problem is that IONM cannot be used during thyroid surgery unless anesthesia is induced by intubating the pa- tient with an EMG endotracheal tube before surgery. In addition, IONM is difficult to use in patients with a tracheostomy tube inserted or in patients with a narrow trachea because of a huge goiter or tumor invasion. Furthermore, EMG endotracheal tubes are relatively expensive; hence, there may be limitations in their coverage by medical insurance.

New types of EMG endotracheal tubes with surface electrodes have been developed to improve contact between the vocal folds and surface electrodes. Further, an alternative IONM system is being developed using adhesive skin electrodes and transcutaneous or transcartilage needle electrodes, which do not require an EMG endotracheal tube [7-10]. Adhesive skin electrodes have the disadvantage of a low amplitude, but they can stably measure the EMG signal of the laryngeal muscle. Transcutaneous or transcartilage needle electrodes can acquire a high amplitude with low false-positive results, but they may be exposed to the surgical field and interfere with the surgical procedure around the upper pole of the thyroid gland. They also have the disadvantage of being a relatively invasive method that can cause bleeding or hematoma in the vocal fold because of needle insertion. However, an IONM system using adhesive skin electrodes and transcutaneous or transcartilage needle electrodes has the advantage of inexpensively measuring the EMG signal of the laryngeal muscle without the EMG endotracheal tube. Furthermore, this technique is advantageous for intraoperative applications, even in patients who have not been administered anesthesia and intubated using the EMG endotracheal tube.

To confirm the state of the RLN by evaluating the movement of the laryngeal muscles, two pairs of electrode must contact the vocal folds. If either electrode has poor contact, accurate EMG measurements cannot be made. Therefore, instead of evaluating muscle movements using EMG, novel IONM systems that mea-

Copyright () 2020 by Korean Society of Otorhinolaryngology-Head and Neck Surgery.

This is an open-access article distributed under the terms of the Creative Commons Attribution Non-Commercial License (https://creativecommons.org/licenses/by-nc/4.0)

which permits unrestricted non-commercial use, distribution, and reproduction in any medium, provided the original work is properly cited. 
sure muscle movements with a new single sensor, such as a surface pressure sensor or an accelerometer based on a piezo-electric sensor, have been introduced [11,12]. In the future, these sensors can be further developed into skin adhesive sensors or endotracheal tube-attached sensors.

It is important to preserve the RLN during thyroid surgery. Several methods and technologies are being developed to overcome the disadvantages of the current IONM system using the EMG endotracheal tube. In the future, we believe that these developments will help surgeons preserve the RLN during thyroid surgery.

\section{CONFLICT OF INTEREST}

No potential conflict of interest relevant to this article was reported.

\section{ORCID}

Sung-Chan Shin https://orcid.org/0000-0003-2329-0648

Byung-Joo Lee https://orcid.org/0000-0001-7091-6688

\section{AUTHOR CONTRIBUTIONS}

Conceptualization: BJL. Data curation: SCS. Formal analysis, Methodology, \& Project administration: BJL. Visualization: SCS. Writing-original draft: all authors. Writing-review \& editing: BJL.

\section{REFERENCES}

1. Bai B, Chen W. Protective Effects of intraoperative nerve monitoring (IONM) for recurrent laryngeal nerve injury in thyroidectomy: me- ta-analysis. Sci Rep. 2018 May;8(1):7761.

2. Shin SC, Lee BJ. Utility of intraoperative neuromonitoring in thyroid surgery. J Clin Otolaryngol. 2018:29(2);157-61.

3. Chiang FY, Lu IC, Chang PY, Sun H, Wang P, Lu XB, et al. Stimulating dissecting instruments during neuromonitoring of RLN in thyroid surgery. Laryngoscope. 2015 Dec;125(12):2832-7.

4. Sung ES, Lee JC, Shin SC, Choi SW, Jung DW, Lee BJ. Development of a novel detachable magnetic nerve stimulator for intraoperative neuromonitoring. World J Surg. 2018 Jan;42(1):137-42.

5. Sung ES, Lee JC, Kim SH, Shin SC, Jung DW, Lee BJ. Development of an attachable endoscopic nerve stimulator for intraoperative neuromonitoring during endoscopic or robotic thyroidectomy. Otolaryngol Head Neck Surg. 2018 Mar;158(3):465-8.

6. Dralle H, Sekulla C, Lorenz K, Brauckhoff M, Machens A, German IONM Study Group. Intraoperative monitoring of the recurrent laryngeal nerve in thyroid surgery. World J Surg. 2008 Jul;32(7):1358-66.

7. Lee HS, Oh J, Kim SW, Jeong YW,Wu CW, Chiang FY, et al. Intraoperative neuromonitoring of recurrent laryngeal nerve during thyroidectomy with adhesive skin electrodes. World J Surg. 2020 Jan; 44(1):148-54.

8. Li P, Liang QZ, Wang DL, Han B, Yi X, Wei W, et al. The transcutaneous electromyography recording method for intraoperative neuromonitoring of recurrent laryngeal nerve during minimally invasive parathyroidectomy. Sci Rep. 2020 May;10(1):7609.

9. Wu CW, Chiang FY, Randolph GW, Dionigi G, Kim HY, Lin YC, et al. Feasibility of intraoperative neuromonitoring during thyroid surgery using transcartilage surface recording electrodes. Thyroid. 2018 Nov;28(11):1508-16.

10. Jung SM,Tae K, Song CM, Lee SH, Jeong JH, JiYB. Efficacy of transcartilaginous electrodes for intraoperative neural monitoring during thyroid surgery. Clin Exp Otorhinolaryngol. 2020 Nov;13(4):422-8.

11. Sung ES, Lee JC, Shin SC, Kwon HK, Na HS, Park DH, et al. Development of a novel intraoperative neuromonitoring system using an accelerometer sensor in thyroid surgery: a porcine model study. Clin Exp Otorhinolaryngol. 2019 Nov;12(4):420-6.

12. Sung ES, Lee JC, Shin SC, Kwon HG, Kim MS, Kim DJ, et al. Development of a novel intraoperative neuromonitoring system using a surface pressure sensor to detect muscle movement: a rabbit model study. Clinical Exp Otorhinolaryngol. 2019 May;12(2):217-23. 